Journal of Applied AnALysis

Vol. 8, No. 1 (2002), pp. 129-139

\title{
THE EXISTENCE AND UNIQUENESS OF SOLUTION OF ONE COUPLED PLATE THERMOMECHANICS PROBLEM
}

\author{
V. A. KRYSKO, J. AWREJCEWICZ and V. M. BRUK \\ Received August 25, 2000 and, in revised form, February 4, 2002
}

\begin{abstract}
The thermo-elastic plate system of equations is analysed. The sufficient conditions of existence, uniqueness and continuity dependence on initial data of the Cauchy problem solutions for differentialoperational equation of mixed type (a part of the equation of hyperbolic type, and a part of parabolic type) are given in this paper. If the operational coefficients are suitably chosen, the investigated equation can be used to obtain a differential equation describing vibrations of a plate the modified Germain-Lagrange equation of hyperbolic type. Moreover, in order to define the temperature field, one can use a three-dimensional equation of thermal conductivity (a parabolic equation).
\end{abstract}

\section{Introduction}

Let $H$ be a Hilbert space, let $L, M$ be a self-adjoint operators negatively defined in $H$ with the domains $D(M) \subset D(L)$, and let $C$ be an operator bounded in $H$. It is assumed that the operator $L$ commutes with $C$ and

2000 Mathematics Subject Classification. 35A07, 74K20, 35J15, 35L10.

Key words and phrases. Thermo-elastic plate, hyperbolic and parabolic equations, vibrations, commutativity.

This work has been supported by the Polish State Committee for Scientific Research (grant No 8T11F 014 16).

ISSN 1425-6908 (C) Heldermann Verlag. 
with the resolvent $M$ (the definition of commuting is given in reference [5]). Let us consider the following system on the interval $[0, T]$

$$
\begin{aligned}
W^{\prime \prime}(t)+L^{2} W(t)+\alpha L C \theta(t) & =q_{1}(t), \\
\theta^{\prime}(t)-M \theta(t)-\beta C^{*} L W^{\prime}(t) & =q_{2}(t),
\end{aligned}
$$

$(\alpha, \beta>0)$ with unknown functions $W$ and $\theta$.

We take $H=L_{2}(\Omega)$ as the space of functions defined in the bounded domain with the piecewise smooth boundary $\partial \Omega$ in the space $\Omega=\Omega_{1} \times$ $[-h / 2, h / 2] \subset \mathbb{R}^{3}\left(\Omega_{1} \subset \mathbb{R}^{2}, h>0\right)$ and having the summing up square norm. As $M$ we take the operator which is realization of the formal differential expression

$$
\frac{\lambda_{0}^{2} \partial^{2}}{\partial x^{2}}+\frac{\lambda_{0}^{2} \partial^{2}}{\partial y^{2}}+\frac{\partial^{2}}{\partial z^{2}} \quad\left(\lambda_{0} \neq 0\right)
$$

and with the attached boundary condition $\left.\theta\right|_{\partial \Omega}=0$. As $L$ operator we take the operator being a closure of a tensor product of the operators $L_{1}$ and $E$, where $L_{1}$ is generated in $L_{2}\left(\Omega_{1}\right)$ by the expression

$$
\gamma \nabla^{2}=\gamma\left(\frac{\partial^{2}}{\partial x^{2}}+\frac{\partial^{2}}{\partial y^{2}}\right) \quad(\gamma>0)
$$

with the boundary condition $\left.W\right|_{\partial \Omega_{1}}=0 . \quad E$ is the identity operator in $L_{2}(-h / 2, h / 2)$, i.e. $L$ is the closure of the operator generated in $L_{2}(\Omega)$ by the expression

$$
\gamma \nabla^{2}=\gamma\left(\frac{\partial^{2}}{\partial x^{2}}+\frac{\partial^{2}}{\partial y^{2}}\right)
$$

and defined on smooth functions from $L_{2}(\Omega)$ which are equal to zero on $\partial \Omega_{1} \times[-h / 2, h / 2]$. The operator $C$ is defined by the formula

$$
C \theta=\int_{-h / 2}^{h / 2} z \theta(x, y, z) d z .
$$

Then

$$
C^{*} g=z \int_{-h / 2}^{h / 2} g(x, y, z) d z .
$$

If $L, M, C$ are chosen in this way, and the numbers $\alpha, \beta, \gamma$ are properly chosen, one can get from (1) the known plate equation governing its vibrations (see, for instance, [1]), with the following parameters: $F=0, k_{x}=k_{y}=0$, $P_{x}=P_{y}=0$; it is called the thermal conductivity equation.

Furthermore, in order to simplify the considerations we assume in (1) that $\alpha=\beta=1$. Observe that practically the introduced considerations do not change other values of $\alpha$ and $\beta$. 
In order to reduce the system (1) into one equation of the first order we introduce the operator

$$
A_{0}=\left(\begin{array}{ccc}
0 & E & 0 \\
-L^{2} & 0 & -L C \\
0 & C^{*} L & M
\end{array}\right),
$$

acting in the space $\widetilde{H}=H \oplus H \oplus H$. Here and further $E$ means the identity operator in the respective space. We emphasize that the commutativity of the operators $C$ and $L$ yields the commutativity of $C^{*}$ and $L^{*}=L[5]$.

Lemma 1. The operator $A_{0}$ allowes of closing of $A$ in the space $\widetilde{H}$. The operator $A^{-1}$ exists, is bounded and defined in the whole space $\widetilde{H}$.

Proof. The operational matrix

$$
\left(\begin{array}{ccc}
C M^{-1} C^{*} & -L^{-2} & -L^{-1} C M^{-1} \\
E & 0 & 0 \\
-L M^{-1} C^{*} & 0 & M^{-1}
\end{array}\right)
$$

defines the bounded operator in $\widetilde{H}$. A direct check shows that its product (of any order) with the operational matrix defining the operator $A_{0}$ gives the unit operational matrix. All claims of the lemma result from this. Moreover, the operator $A^{-1}$ is defined by the last operational matrix.

Further on, the operator and its closure will be tagged with one symbol. If we assume $\nu=w^{\prime}$, and $\mathrm{u}$ will denote the column

$$
\left(\begin{array}{l}
w \\
\nu \\
\theta
\end{array}\right)
$$

then the homogeneous system (1) in the space $\widetilde{H}$ can be written in the following way:

$$
u^{\prime}(t)=A u(t)
$$

Furthermore, we denote by $\widetilde{L}^{-2}$ the operator in $\widetilde{H}$, defined by the equation

$$
\widetilde{L}^{-2}=\left(\begin{array}{ccc}
L^{-2} & 0 & 0 \\
0 & L^{-2} & 0 \\
0 & 0 & L^{-2}
\end{array}\right) .
$$

It results from the commuting properties of the operators that also $\widetilde{L}^{-2}$ and $A^{-1}$ commute. 


\section{Basic assumption}

In order to formulate the basic assumption we need to define the following spaces and the attached operators. The smoothness of a solution to the homogeneous equation (2) depends on that if the initial condition $u(0)$ belongs to the domains of the operators $\widetilde{L}$ and $A$. Observe that in applications the operator $\widetilde{L}$ is simpler than the operator $A$ and therefore the spaces are constructed with regard to the powers of $\widetilde{L}$. However, we can not completely omit the operator $A$. Observe also that one can use the powers of $A$ only (without $\widetilde{L}$ ).

We define the scalar product by the formula $(x, y)_{F^{*}}=\left(A^{*} x, A^{*} y\right)$ on the manifold $F^{*}=D\left(A^{*}\right)$. As the operator $A^{*}$ possesses the inverse one defined everywhere, then $F^{*}$ is a complete space and $\|x\|_{F^{*}} \geq\|x\|_{\widetilde{H}}$. This is why $F^{*}$ can be considered as the space with a positive norm in relation to $\widetilde{H}$ [2]. We shall denote by $\widetilde{H}^{(-1)}$ the space with a negative norm, constructed using the pair $F^{*}, \widetilde{H}$.

The operator $A^{*}$ maps continuously and bijectively $F^{*}$ into $\widetilde{H}$. This is why the conjugated operator $\widetilde{A}$ acts from $\widetilde{H}$ to $\widetilde{H}^{(-1)}$ continuously and bijectively, and it serves as the extension of $A$.

We shall define the scalar product on the set $\widetilde{H}^{(+1)}=D(A)$ by the equation $(x, y)_{\widetilde{H}^{(+1)}}=(A x, A y) . \widetilde{H}^{(+1)}$ appears as the full space and the operator $A$ maps $\widetilde{H}^{(+1)}$ to $\widetilde{H}$ continuously and bijectively (one-to-one).

Because the operator $\widetilde{L}^{-1}$ commutes with $A, A^{*}$, and $\widetilde{L}^{-1}$ is self-adjoint and bounded in $\widetilde{H}$, then $\widetilde{L}^{-1}$ is self-adjoint and bounded in the spaces $\widetilde{H}^{(+1)}$ and $\widetilde{H}^{(-1)}$ (the $\widetilde{L}^{-1}$ operator is extended due to continuity in the space $\left.\widetilde{H}^{-1}\right)$. The Hilbert space scales will be denoted by the symbols $\widetilde{H}_{\alpha}^{(+1)}$, $\widetilde{H}_{\alpha}=\widetilde{H}_{\alpha}^{0}, \widetilde{H}_{\alpha}^{(-1)}(-\infty<\alpha<\infty)[2]$, and they are generated by the operator $\widetilde{L}$ acting in $\widetilde{H}^{(+1)}, \widetilde{H}$ and $\widetilde{H}^{(-1)}$, correspondingly. As it is known [2] for $\alpha \geq 0$ the operator $\widetilde{L}$ maps $\widetilde{H}_{\alpha+1}^{(\beta)}$ into $\widetilde{H}_{\alpha}^{(\beta)}(\beta=-1,0,1)$ continuously and bijectively, and $\widetilde{L}$ allows for continuous and bijective extension from $\widetilde{H}_{-\alpha}^{(\beta)}$ to $\widetilde{H}_{-\alpha-1}^{(\beta)}(\alpha \geq 0)$. We shall denote this extension as $\widetilde{L}$ in order not to introduce redundant denotations.

\section{Main results}

The operators $A, \widetilde{A}$ map $\widetilde{H}_{\alpha}^{(+1)}$ into $\widetilde{H}_{\alpha}$, and $\widetilde{H}_{\alpha}$ into $\widetilde{H}_{\alpha}^{(-1)}(\alpha \geq 0)$ continuously and bijectively, respectively. The same theorem is true for $\alpha<0$, and for the operators $\widetilde{L}^{\alpha} A \widetilde{L}^{-\alpha}$ and $\widetilde{L}^{\alpha} \widetilde{A} \widetilde{L}^{-\alpha}$, which serve as the 
extension of $A$ and $\widetilde{A}$, respectively. These extensions are also denoted by $A$ and $\widetilde{A}$.

Theorem 1. For any element $u_{0} \in \widetilde{H}_{\alpha}^{(\beta)}(\beta=+1,0 ;-\infty<\alpha<\infty)$ there exists one function $u(t)$ continuous in the closed interval $[0, T]$ in the space $\widetilde{H}_{\alpha-2}^{(\beta-1)}$, continuous in the half open from the left side interval $(0, T]$ in $\widetilde{H}_{\alpha-4}^{(\beta)}$, differentiable on $(0, T]$ in $\widetilde{H}_{\alpha-4}^{(\beta-1)}$ and satisfying both the equation

$$
u^{\prime}(t)=\widetilde{A} u(t),
$$

and the condition $u(0)=u_{0}$. (The continuity is understood in the strong sense in a suitable space.)

Observe that from the formulated theorem one can conclude that, in particular, for $u_{0} \in \widetilde{H}_{4}^{(+1)}$ the function $u(t)$ is a weak solution of the equation (2) (in the sense given in reference [3]).

The proof of Theorem 1 is based on the quoted below estimates of the resolvent of the operator $A$ in a way similar to that presented in [3], and the Laplace inverse transformation.

Let $\lambda=\sigma+i \tau$. We define the operators $\triangle_{1}=\triangle_{1}(\lambda), \triangle_{2}=\triangle_{2}(\lambda)$ in $H$ in the following way:

$$
\begin{aligned}
& \triangle_{1}=\left(\lambda^{2} E+L^{2}\right)-\lambda L^{2} C(M-\lambda E)^{-1} C^{*}, \\
& \triangle_{2}=(M-\lambda E)-\lambda L^{2}\left(L^{2}+\lambda^{2} E\right)^{-1} C^{*} C .
\end{aligned}
$$

Lemma 2. If $\sigma=\operatorname{Re} \lambda>0$ then the operators $\triangle_{1}, \triangle_{2}$ have bounded inverses:

$$
\left\|\triangle_{1}^{-1}\right\| \leq \frac{1}{\sigma|\lambda|}, \quad\left\|\triangle_{2}^{-1}\right\| \leq \frac{1}{\sigma}
$$

Proof. Let $S=(1 / \lambda) \triangle_{1}$. Then for any $f \in D\left(L^{2}\right)$ we have:

$$
\begin{aligned}
(S f, f)= & (\sigma+i \tau)(f, f)+\frac{1}{|\lambda|^{2}}(\sigma-i \tau)\left(L^{2} f, f\right) \\
& -\left((M-\sigma E+i \tau E)|M-\lambda E|^{-1} L C^{*} f, \quad|M-\lambda E|^{-1} L C^{*} f\right) .
\end{aligned}
$$

It results from this and from the negative definition of $M$ that $\operatorname{Re}(S f, f) \geq$ $\sigma\|f\|^{2}$, and therefore $\|S f\| \geq \sigma\|f\|$. Analogously we get $\left\|S^{*} g\right\| \geq \sigma \|$ $g \|$. This is why the operator $S^{-1}$ exists, is bounded, defined on the whole $H$, and $\left\|S^{-1}\right\| \leq 1 / \sigma$.

The equation

$$
-\lambda L^{2}\left(L^{2}+\lambda^{2} E\right)^{-1}=-L^{2}\left(\lambda L^{2}+|\lambda|^{2} \lambda E\right)\left|L^{2}+\lambda^{2} E\right|^{-2}
$$


yields $\operatorname{Re}\left(-\lambda L^{2}\left(L^{2}+\lambda^{2} E\right)^{-1} C^{*} C g, g\right) \leq 0(g \in H)$. From the above and taking into account the negative definition of the operator $M$ we get: $\operatorname{Re}\left(\triangle_{2} f, f\right) \leq-\sigma(f, f)\left(f \in D\left(\triangle_{2}\right)\right)$. This is why $\left|\operatorname{Re}\left(\triangle_{2} f, f\right)\right| \geq \sigma\|f\|^{2}$, which yields $\left\|\triangle_{2} f\right\| \geq \sigma\|f\|$. Analogously, we get $\left\|\triangle_{2}^{*} f\right\| \geq \sigma\|f\|$. Two last inequalities prove the lemma on the operator $\triangle_{2}$.

Lemma 3. If $\operatorname{Re} \lambda \geq 0$ then the operator $A$ possesses the resolvent $R(\lambda)$ which is defined by the matrix

$$
\begin{aligned}
& R(\lambda)= \\
& \left(\begin{array}{ccc}
-\lambda \triangle_{1}^{-1}+L^{2} \triangle_{1}^{-1} C(M-\lambda E)^{-1} C^{*} & -\triangle_{1}^{-1} & -\triangle_{1}^{-1} L C(M-\lambda E)^{-1} \\
L^{2} \triangle_{1}^{-1} & -\lambda \triangle_{1}^{-1} & -\lambda \triangle_{1}^{-1} L C(M-\lambda E)^{-1} \\
-L^{3}\left(L^{2}+\lambda^{2} E\right)^{-1} \triangle_{2}^{-1} C^{*} & \lambda L\left(L^{2}+\lambda^{2} E\right)^{-1} \triangle_{2}^{-1} C^{*} & \triangle_{2}^{-1}
\end{array}\right) .
\end{aligned}
$$

Proof. By direct check and taking into account the commutativity of $L$ and $M^{-1}$ one can verify that the expression $R(\lambda)\left(A_{0}-\lambda E\right)$ gives a unit operator matrix. Besides, the space of values of the operator $A_{0}-\lambda E$ is dense in $\widetilde{H}$. Indeed, let such the element $x=\left(x_{1}, x_{2}, x_{3}\right) \in \widetilde{H}$ occurs, that $\left(A_{0}-\lambda E\right)^{*} x=0$. This equation is equivalent to the following three:

$-\bar{\lambda} x_{1}-L^{2} x_{2}=0, \quad x_{1}-\bar{\lambda} x_{2}+C L x_{3}=0, \quad-C^{*} L x_{2}+(M-\bar{\lambda} E) x_{3}=0$.

Finding $x_{1}$ from the first equation, $x_{3}$ from the last one and by substituting in the second one, we get $\triangle_{1}(\bar{\lambda}) x_{2}=0$. It results from Lemma 2 that $x_{2}=0$, and therefore $x_{1}=x_{3}=0$. This is why $x=0$.

Lemma 4. If $\sigma=\operatorname{Re} \lambda>0$ are large enough the following estimations hold

$$
\begin{aligned}
& \|R(\lambda)\| \leq k \frac{|\lambda|}{\sigma}, \\
& \left\|R(\lambda) \widetilde{L}^{-2}\right\| \leq k \frac{1}{|\lambda|} .
\end{aligned}
$$

Here and further $k$ denotes constant (independent of $\lambda$ ), but different, generally speaking, for different inequalities.

Proof. First we are going to prove the inequality

$$
\left\|L^{2} \triangle_{1}^{-1}\right\| \leq \frac{|\lambda|}{\sigma} .
$$

As in the proof of Lemma 2, if $g \in H$, the following holds

$$
\begin{aligned}
\left(S L^{-2} g, g\right)= & (\sigma+i \tau)\left(L^{-2} g, g\right)+\frac{1}{|\lambda|^{2}}(\sigma-i \tau)(g, g) \\
& -\left((M-\sigma E-i \tau E)|M-\lambda E|^{-1} C^{*} g,|M-\lambda E|^{-1} C^{*} g\right) .
\end{aligned}
$$


Then $\operatorname{Re}\left(S L^{-2} g, g\right) \geq \sigma /|\lambda|^{2}$. Thus (as in Lemma 2) we see that $\left\|L^{2} S^{-1}\right\| \leq|\lambda|^{2} / \sigma$ and this is why (6) is satisfied.

Let us denote the elements of operator matrix defining $R(\lambda)$ by $a_{i j}$. Then:

$$
\begin{array}{lll}
\left\|a_{11}\right\| \leq \frac{k}{\sigma}, & \left\|a_{12}\right\| \leq \frac{1}{|\lambda| \sigma}, & \left\|a_{13}\right\| \leq \frac{k}{|\lambda| \sigma}, \\
\left\|a_{21}\right\| \leq \frac{|\lambda|}{\sigma}, & \left\|a_{22}\right\| \leq \frac{1}{\sigma}, & \left\|a_{23}\right\| \leq \frac{k}{\sigma}, \\
\left\|a_{11} L^{-2}\right\| \leq \frac{k}{|\lambda|}, & \left\|a_{12} L^{-2}\right\| \leq \frac{k}{|\lambda| \sigma}, & \left\|a_{13} L^{-2}\right\| \leq \frac{k}{|\lambda|^{2} \sigma}, \\
\left\|a_{21} L^{-2}\right\| \leq \frac{1}{|\lambda| \sigma}, & \left\|a_{22} L^{-2}\right\| \leq \frac{k}{|\lambda|}, & \left\|a_{23} L^{-2}\right\| \leq \frac{k}{|\lambda| \sigma} .
\end{array}
$$

The estimations of elements of the first two rows of the matrix $R(\lambda)$ immediately result from (6) of Lemma 2, the inequalities $\left\|L(M-\lambda E)^{-1}\right\| \leq k$, and

$$
\left\|(M-\lambda E)^{-1}\right\| \leq \frac{1}{|\lambda|},
$$

and also from the following two expressions

$a_{11}=-\frac{1}{\lambda} E-\frac{1}{\lambda} L^{2} \triangle_{1}^{-1}, \quad a_{22} L^{-2}=-\frac{1}{\lambda} L^{-2}+\frac{1}{\lambda} \triangle_{1}^{-1}+\triangle_{1}^{-1} C(M-\lambda E)^{-1} C^{*}$, which can be veryfied using the elementary transformations.

In order to evaluate the elements of the third row of the matrix $R(\lambda)$ we shall add some supporting inequalities.

Because for any $f \in D\left(L^{2}\right)$ we have $\operatorname{Re}\left(\left(L^{2} / \lambda+\lambda E\right) f, f\right) \geq \sigma(f, f)$, then $\left\|\left(L^{2} / \lambda+\lambda E\right)^{-1}\right\| \leq 1 / \sigma$. This yields

$$
\left\|\left(L^{2}+\lambda^{2} E\right)^{-1}\right\| \leq \frac{1}{|\lambda| \sigma} .
$$

From this inequality (using the elementary transformations) we get

$$
\left\|L^{2}\left(L^{2}+\lambda^{2} E\right)^{-1}\right\| \leq \frac{|\lambda|}{\sigma} .
$$

We shall prove that

$$
\left\|L \triangle_{2}^{-1}\right\| \leq m
$$

where $m$ does not depend on $\lambda$. For any $f \in D\left(\triangle_{2} L^{-1}\right)$ we have

$$
\begin{aligned}
\left(\triangle_{2} L^{-1} f, f\right)= & \left(M L^{-1} f, f\right)-(\sigma+i \tau)\left(L^{-1} f, f\right)-(\sigma-i \tau)|\lambda|^{2}(L g, g) \\
& -(\sigma+i \tau)\left(L^{3} g, g\right),
\end{aligned}
$$

where $g=\sqrt{\left|L^{2}+\lambda^{2} E\right|^{-2} C^{*} C f}$. It results from the negative definition of $L$ that

$$
\operatorname{Re}\left(\triangle_{2} L^{-1} f, f\right) \geq \operatorname{Re}\left(M L^{-1} f, f\right) \geq k\|f\|^{2} .
$$


The last inequality results from the positive definition of the limited operator $L M^{-1}$, inversed to $M L^{-1}$. Therefore, the inequality (10) has been satisfied. The equation

$$
\triangle_{2}^{-1} L^{-1}=(M-\lambda E)^{-1} L^{-1}+\left(L \triangle_{2}^{-1}\right)\left(L^{2}+\lambda^{2} E\right)^{-1} C^{*} C \lambda(M-\lambda E)^{-1}
$$

and the inequalities (7), (8), (10) yield

$$
\left\|\triangle_{2}^{-1} L^{-1}\right\| \leq \frac{k}{|\lambda|}
$$

Now the inequalities (8), (9), (10), (11) and Lemma 2 allow to obtain the estimation of the elements of the third row of the matrix $R(\lambda)$ :

$$
\begin{aligned}
& \left\|a_{31}\right\| \leq k \frac{|\lambda|}{\sigma}, \quad\left\|a_{32}\right\| \leq \frac{k}{\sigma}, \quad\left\|a_{33}\right\| \leq \frac{1}{\sigma}, \\
& \left\|a_{31} L^{-2}\right\| \leq \frac{k}{|\lambda| \sigma}, \quad\left\|a_{32} L^{-2}\right\| \leq \frac{k}{|\lambda| \sigma}, \quad\left\|a_{33} L^{-2}\right\| \leq \frac{k}{|\lambda|} .
\end{aligned}
$$

The proof of Lemma results from the obtained $a_{i j}$ estimates.

Let us notice that some evaluations can be even more improved, for instance $\left\|a_{12} L^{-2}\right\| \leq k /|\lambda|^{2}$. However, they will be not used further.

Now we prove directly the theorem. Following reference [3] we are looking for the solution of the equation (2) in the form

$$
u(t)=-\frac{1}{2 \pi i} \int_{\sigma-i \infty}^{\sigma+i \infty} e^{\lambda t} R(\lambda) u(0) d \lambda,
$$

where the integration takes place along the straight line, parallel to imaginary axis and passing through the point $(\sigma, 0)$, where $\sigma>0$ is sufficiently large.

We assume first that $u_{0} \in \widetilde{H}_{4}^{(+1)}$, i.e. $u_{0}=R(0) \widetilde{L}^{-4} z_{0}\left(z_{0} \in \widetilde{H}\right)$, and we consider the function

$$
\begin{aligned}
u(t) & =-\frac{1}{2 \pi i} \int_{\sigma-i \infty}^{\sigma+i \infty} e^{\lambda t} R(\lambda) R(0) \widetilde{L}^{-4} z_{0} d \lambda \\
& =-\frac{1}{2 \pi i} \int_{\sigma-i \infty}^{\sigma+i \infty} e^{\lambda t}\left(\frac{1}{\lambda} R(\lambda) \widetilde{L}^{-4} z_{0}-\frac{1}{\lambda} R(0) \widetilde{L}^{-4} z_{0}\right) d \lambda .
\end{aligned}
$$

Here, while passing from the first integral to the second one, the resolvent identity has been used. The existence of the integral, the continuity of $u(t)$ on $[0, \mathrm{~T}]$ in the space $\widetilde{H}_{+2}$ (and correspondingly, in $\widetilde{H}$ ) and the identity $u(0)=R(0) \widetilde{L}^{-4} z_{0}$ result directly from the Lemma 4 . 
Applying the integration by parts to the first integral in (12) and taking into consideration Lemma 4 we get

$$
u(t)=\frac{1}{2 \pi i} \frac{1}{t} \int_{\sigma-i \infty}^{\sigma+i \infty} e^{\lambda t} R^{2}(\lambda) R(0) \widetilde{L}^{-4} z_{0} d \lambda .
$$

It results from the above that $u(t)$ is continuous on $(0, \mathrm{~T}]$ in the space $\widetilde{H}^{(+1)}$ and it possesses the continuous derivative on $(0, \mathrm{~T}]$ in $\widetilde{H}$

$$
\begin{aligned}
u^{\prime}(t)= & -\frac{1}{2 \pi i} \frac{1}{t^{2}} \int_{\sigma-i \infty}^{\sigma+i \infty} e^{\lambda t} R^{2}(\lambda) R(0) \widetilde{L}^{-4} z_{0} d \lambda \\
& +\frac{1}{2 \pi i} \frac{1}{t} \int_{\sigma-i \infty}^{\sigma+i \infty} e^{\lambda t} \lambda R^{2}(\lambda) R(0) \widetilde{L}^{-4} z_{0} d \lambda,
\end{aligned}
$$

and satisfies the equation (2).

The uniqueness of the solution of the Cauchy problem for the equation (2) follows from the estimation (4) and the results presented in reference [3].

Now, let $u_{0} \in \widetilde{H}_{\alpha}^{(\beta)}(\beta=+1,0 ;-\infty<\alpha<\infty)$. Then $x_{0}=A^{\beta-1} \widetilde{L}^{\alpha-4} u_{0} \in$ $\widetilde{H}_{4}^{(+1)}$ (here $A^{0}$ denotes $E$ ). Because the operators $R(\lambda)$ and $\widetilde{L}^{\alpha}$ commute and from the previous considerations we get the function

$$
u(t)=-\frac{1}{2 \pi i} \widetilde{A}^{1-\beta} \widetilde{L}^{4-\alpha} \int_{\sigma-i \infty}^{\sigma+i \infty} e^{\lambda t} R(\lambda) x_{0} d \lambda
$$

with all properties mentioned in the formulated theorem. The uniqueness of such function results from the fact that the function $A^{\beta-1} \widetilde{L}^{\alpha-4} u(t)$ is the solution of the equation (2). Theorem 1 has been proved.

Remark 1. It results from the above proof that in order to solve the equations (2) and (3), the estimation $\|u(t)\|_{\widetilde{H}_{\alpha}^{(\beta-1)}} \leq k\|u(0)\|_{\widetilde{H}_{\alpha+2}^{(\beta)}}$ $(\beta=+1,0 ;-\infty<\alpha<\infty)$ holds, where $k$ does not depend on $t$. It defines the continuous dependence of solution on the initial data in the corresponding spaces.

Let us pass to the consideration of the non-homogeneous equations

$$
u^{\prime}(t)=\widetilde{A} u(t)+f(t) .
$$


By $U(t)$ we denote the operator

$$
U(t) x=-\frac{1}{2 \pi i} \int_{\sigma-i \infty}^{\sigma+i \infty} e^{\lambda t} R(\lambda) x d \lambda, \quad 0 \leq t \leq T
$$

defined for all $x$, for which the last integral exists. The Lemma 4 and the equation

$$
U(t) R(0) \widetilde{L}^{-2} z=-\frac{1}{2 \pi i} \int_{\sigma-i \infty}^{\sigma+i \infty} e^{\lambda t} \frac{1}{\lambda}(R(\lambda)-R(0)) \widetilde{L}^{-2} z d \lambda
$$

imply that for every fixed $t$, the operator $U(t): \widetilde{H}_{\alpha}^{(\beta)} \rightarrow \widetilde{H}_{\alpha-2}^{(\beta-1)}(\beta=$ $+1,0 ;-\infty<\alpha<\infty)$ is bounded and the operator function $U(t)$ is strongly continuous on $[0, T]$. For any $u_{0} \in \widetilde{H}_{\alpha}^{(\beta)}$, the function $u(t)=U(t) u_{0}$ has the properties given in Theorem 1 .

Theorem 2. Let for any $t \in[0, T], f(t)$ belongs to the domain of the operator $\widetilde{L}^{\alpha} A^{1+\beta}(\beta=+1,0 ;-\infty<\alpha<\infty)$ and let the function $\widetilde{L}^{\alpha} A^{1+\beta} f(t)$ be continuous in $\widetilde{H}$. Then the function

$$
u(t)=\int_{0}^{t} U(t-s) f(s) d s
$$

is continuous on $[0, T]$ in the space $\widetilde{H}_{\alpha-2}^{(\beta)}$, is differentiable on $[0, T]$ in the space $\widetilde{H}_{\alpha-2}^{(\beta-1)}$ and satisfies the equation (13).

Proof. Let $g(t)=\widetilde{L}^{\alpha} A^{1+\beta} f(t)$. Following the steps given in the reference [6] one can conclude that the function (14) and its derivative have the form

$$
\begin{aligned}
u(t) & =\int_{0}^{t} U(t-s) A^{-1-\beta} \widetilde{L}^{-\alpha} g(s) d s \\
u^{\prime}(t) & =A^{-1-\beta} \widetilde{L}^{-\alpha} g(t)+\int_{0}^{t} U_{t}^{\prime}(t-s) A^{-1-\beta} \widetilde{L}^{-\alpha} g(s) d s \\
& =f(t)+\int_{0}^{t} \widetilde{A} U(t-s) A^{-1-\beta} \widetilde{L}^{-\alpha} g(s) d s=f(t)+\widetilde{A} u(t) .
\end{aligned}
$$

The existence of integrals in these equations results from the continuity of $g$ and the properties of $U(t)$. In what follows $u(t)$ is the solution of the equation (13). The theorem has been proved.

Remark 2. The continuity condition of the function $\widetilde{L}^{\alpha} A^{1+\beta}$ can be weakened if the function $f$ is smooth enough. 
Finally, we acknowledge the recent developments of a similar problem considered in our work pointed out by one of the reviewers and presented in references [6], [4] (see also the extended bibliography therein). However, in the mentioned works there is no non-local term $C$ but there appears a mixed fourth order term accounting for the rotational forces. In addition, in reference [6] the stability properties are considered using the boundary feedback modifications, which are not considered in our work. In the works of Lasiecka (see [4] and the cited literature therein) the asymptotic behaviour governed by von Kármán equations is analysed which is out of the content of our paper.

Acknowledgements. The authors thank the reviewers for their proper remarks and comments.

\section{References}

[1] Awrejcewicz, J., Krysko, V. A., Numerical Analysis of Shells with Thermal Load (in Polish), Scientific-Technical Publisher WNT, Warsaw, 1998.

[2] Berezanskij, Yu. M., Expansions in Eigenfunctions of Self-Adjoint Operators (in Russian), Naukova Dumka, Kiev, 1965, Amer. Math. Soc. Transl. Ser. 2 17, Providence, RI, 1968.

[3] Krein, S. G., Linear Differential Equations in Banach Spaces (in Russian), Nauka, Moskva, 1967.

[4] Lasiecka, I., Finite dimensionality and compactness of attractor for von Kármán equations with nonlinear dissipation, Nonlinear Differential Equations Appl. 6 (1999), $337-472$.

[5] Riesz, F., Szökefalvi-Nagy, B., Lectures on Functional Analysis, Undaw, New York, 1995.

[6] Triggiani, R., Improvent of stability properties of hyperbolic damped wave equation via boundary feedback, Lecture Notes in Control and Inform. Sci. 75 (1985), Springer, Berlin, 400-409.

J. AWREJCEWICZ

Department of Automatics

AND BIOMECHANICS

TECHNICAL UnIVERSITY OF LÓDŹ

1/15 Stefanowski St.

90-924 LóDź, POLAND

E-MAIL:AWREJCEW@CK-SG.P.LODZ.PL
V. A. Krysko and V. M. Bruk

DEPARTMENT OF MATHEMATICS

TeChnical University of SARATOV

41005 Saratov, Russia 